\title{
Politique
}

Politique

\section{Philippe Faucher et Johanne Bergeron, Hydro-Québec, la société de l'heure de pointe, Montréal, Les Presses de l’Université de Montréal, 1986, 221 p.}

\section{Jeanne Kirk Laux}

Numéro 11, hiver 1987

L’État privé

URI : https://id.erudit.org/iderudit/040553ar

DOI : https://doi.org/10.7202/040553ar

Aller au sommaire du numéro

Éditeur(s)

Société québécoise de science politique

ISSN

0711-608X (imprimé)

1918-6584 (numérique)

Découvrir la revue

Citer ce compte rendu

Laux, J. K. (1987). Compte rendu de [Philippe Faucher et Johanne Bergeron,

Hydro-Québec, la société de l'heure de pointe, Montréal, Les Presses de

l’Université de Montréal, 1986, 221 p.] Politique, (11), 163-166.

https://doi.org/10.7202/040553ar

Ce document est protégé par la loi sur le droit d'auteur. L'utilisation des services d'Érudit (y compris la reproduction) est assujettie à sa politique d'utilisation que vous pouvez consulter en ligne.

https://apropos.erudit.org/fr/usagers/politique-dutilisation/ 
Philippe Faucher et Johanne Bergeron, Hydro-Québec, la société de l'heure de pointe, Montréal, Les Presses de l'Université de Montréal, 1986, $221 \mathrm{p}$.

Voici un ouvrage d'une grande qualité qui cherche, et qui réussit, à provoquer une réflexion sur notre mode de développement capitaliste en crise - l'État providence - par une soigneuse remise en question d'une forme d'interventionnisme privilégiée au Québec, l'entreprise publique. Hydro-Québec est revêtu dans ce livre d'une fonction allégorique pour permettre tant la critique de la gestion du secteur public jusqu'à date qu'une incitation au lancement d'une véritable politique industrielle.

Afin d'évaluer la performance d'Hydro-Québec, le livre est divisé en deux parties. Tout d'abord, les auteurs examinent son premier mandat, celui de fournir l'électricité. Après un rappel de l'historique de la nationalisation en insistant sur son caractère tronqué (vue l'auto-approvisionnement de certaines grandes entreprises privées) Faucher et Bergeron exposent les confusions 
entourant les politiques énergétiques (chapitre 5). Dans les trois chapitres consacrés aux grands projets, aux prévisions, et aux exportations ils arrivent à une même conclusion - que ce sont les initiatives ou réticences de la société d'État elle-même qui, jusqu'en 1978, ont orienté ce secteur critique de l'économie. Dans la deuxième partie de l'étude, les auteurs soumettent la gestion de la société d'État à un examen rigoureux pour voir si elle a rempli son deuxième mandat, celui de «faire bénéficier la population du Québec des effets d'entraînements associés avec la production et la distribution d'électricité.» (p. 16).

Il s'agit avant tout de la documentation d'une déception. Vue la mission dont Faucher et Bergeron chargent le secteur public - de servir comme instrument de politique économique pour «bâtir le Québec» - leur bilan des réalisations d'HydroQuébec depuis 1963 est négatif. Bien sûr, cette société d'État a merveilleusement satisfait à son premier mandat, celui de fournir l'électricité aux consommateurs à un prix raisonnable. Mais c'est la contribution d'Hydro-Québec à la réalisation d'objectifs socioéconomiques qui préoccupent surtout les auteurs. Après tout, «si les tarifs, la politique financière et la politique d'achat pratiqués par Hydro-Québec ne se distinguent en rien de ce que ferait une entreprise privée placée dans une situation de marché identique, alors à quoi bon nationaliser?» (p. 122) Leurs analyses scrupuleuses, solidement ancrées dans des documents de l'entreprise ou gouvernementaux, de ces trois aires d'activités leur font conclure que: «Hydro-Québec se comporte pour l'essentiel comme une entreprise privée. » (p. 204).

Comment l'expliquer? Les auteurs sont à la fois très clairs et très sévères. «L'irresponsabilité» des gouvernements incapables de formuler des politiques énergétiques ou industrielles cohérentes (ici sont condamnés tant le Parti libéral que le Parti québécois) conjuguée au "laxisme dans la gestion» d'Hydro-Québec a eu pour résultante un mauvais usage des ressources nationales. C'est 
pourtant à l'État qu'en incombe la responsabilité primordiale. Pourquoi? Selon les théories sur le comportement des entreprises publiques, telles que synthétisées par les auteurs dans l'excellent essai qui constitue le premier chapitre du livre, on ne peut qu'agréer avec l'hypothèse que plus l'indétermination de l'État en matière de politique est grande, plus l'entreprise publique imposera sa propre stratégie, axée sur ses objectifs d'autonomie, de croissance et de stabilité. La portée de sa stratégie dépend de plusieurs facteurs, telle la capacité d'auto-financement de l'entreprise publique. Néanmoins, de façon générale - et ici l'étude de Faucher et Bergeron confirme celles de Hafsi (Québec), Feigenbaum (ÉtatsUnis), Aharoni (Israël) ou Anastassopoulos (France) - «l'entreprise va accroître la distance qui la sépare de l'administration centrale et simultanément elle va tenter de se rapprocher du secteur privé. » (p. 28).

Les auteurs doivent être félicités pour avoir réussi à confronter les lecteurs à des questions souvent techniques - telles les méthodes de prévision de la demande de l'électricité ou des ratios financiers qui soutendent la logique des emprunts - sans pour autant décourager les non-initiés, ceci grâce à des définitions limpides et à un très bon lexique.

Tout au long du texte, et souvent de façon passionnelle dans les conclusions, Faucher et Bergeron présentent un plaidoyer pour un interventionnisme de l'État qui transcenderait les querelles interministérielles et résisterait aux partisans néo-libéraux d'un moins-d'État. Ce n'est pas en invoquant le slogan d'État catalyseur au lieu de celui d'État entrepreneur que nous allons faire face au besoin impératif de trouver un mode de développement collectif capable d'assurer aussi bien une autosuffisance énergétique qu'une base technologique capable de soutenir des industries compétitives. Le véritable mandat d'Hydro-Québec serait donc de jouer le rôle d'entraînement de la croissance, une croissance à base de la technologie et du savoir faire québécois. Ainsi sont tournés en dérision 
les propos de M. Bourassa qui mise sur les exportations sans les avoir liées à une stratégie industrielle sérieuse.

Le livre de Faucher et Bergeron soulèvera sans doute des débats. Des politicologues se demanderont si les auteurs font les naifs: une fois si bien démontrés les blocages des politiques économiques des gouvernements en principe dédiés à un dirigisme d'État (et ceci dans de conditions plus favorables de croissance), comment pourront-ils cette fois accepter les exhortations qui leurs sont adressées à nouveau? Une analyse plus profonde des contradictions socio-économiques qui soutendent l'éclatement de l'appareil de l'État capitaliste serait ici nécessaire pour savoir par où entamer une action politique efficace. Des écologistes pourraient s'objecter à l'appel à une relance des investissements d'Hydro-Québec pour stimuler le développement technologique en dépit des surplus d'énergie. D'autres trouveront que les auteurs exagèrent l'impact que peut avoir une société d'État. Nonobstant sa politique d'achat manifestement préférentielle (selon des donnés ici présentées) le rôle d'Hydro-Québec est déprécié à cause du peu d'amélioration de valeur ajouté manufacturière qu'elle entraîne - l'entreprise est jugée largement responsable de l'absence de producteurs québécois dans l'industrie d'équipement électrique.

La lecture du livre de Faucher et Bergeron est fortement conseillée. Ils nous lancent le défi de poussèr plus loin le débat sur leur conclusion globale: "Disposer d'un secteur public de l'importance de celui qui existe au Québec et ne pas prendre les moyens de le diriger en fonction de priorités de développement propres à la Province est non seulement un vaste gaspillage de ressources mais relève d'une irresponsabilité d'autant plus flagrante qu'il s'agit d'un instrument d'intervention économique puissant qui échappe au contrôle du gouvernement fédéral.» (p. 206)

Jeanne Kirk Laux

Université d'Ottawa 